\title{
METHOD OF LOWER AND UPPER FUNCTIONS IN IMPULSIVE PERIODIC BOUNDARY VALUE PROBLEMS
}

\author{
I. RACHŮNKOVÁ * \\ Department of Mathematics, Palacký University, \\ 77900 OLOMOUC, Tomkova 40, Czech Republic, \\ E-mail: rachunko@risc.upol.cz
}

\section{TVRDÝ ${ }^{\dagger}$}

Mathematical Institute, Academy of Sciences of the Czech Republic, 11567 PRAHA 1, Žitná 25, Czech Republic,

E-mail: turdy@math.cas.cz

\begin{abstract}
We present here new existence results for the nonlinear second order impulsive periodic boundary value problem. They rely on the presence of a pair of associated lower/upper functions. In contrast to the results known up to now, we need not assume that they are well-ordered.
\end{abstract}

\section{Introduction}

Here we present an account on our new existence results for the impulsive periodic boundary value problem

$$
\begin{aligned}
& u^{\prime \prime}=f\left(t, u, u^{\prime}\right), \\
& u\left(t_{i}+\right)=\mathrm{J}_{i}\left(u\left(t_{i}\right)\right) u^{\prime}\left(t_{i}+\right)=\mathrm{M}_{i}\left(u^{\prime}\left(t_{i}\right)\right), i=1,2, \ldots, p, \\
& u(0)=u(T), u^{\prime}(0)=u^{\prime}(T),
\end{aligned}
$$

where

$$
\begin{aligned}
& 0<t_{1}<\cdots<t_{p}<T<\infty, \mathrm{J}_{i}, \mathrm{M}_{i} \in \mathbb{C}(\mathbb{R}), i=1,2, \ldots, p, \\
& f \text { fulfils the Carathéodory conditions on }[0, T] \times \mathbb{R}^{2},
\end{aligned}
$$

Starting with $\mathrm{Hu}$ and Lakshmikantham [5], periodic boundary value problems for nonlinear second order impulsive differential equations of the form

\footnotetext{
*Supported by the grant no. 201/01/1451 of the GAČR and by the Council of Czech Gov. j14/98:153100011

†Supported by grant no. 201/01/1199 of the GAČR
} 
(1.1)-(1.3) have been studied by several authors. A rather representative (however not complete) list of related papers is given in references. In particular, in [1], [2], [4], [6], [7] the existence results in terms of lower/upper functions obtained by the monotone iterative method can be found. All of these results impose monotonicity of $\mathrm{J}_{i}$ and $\mathrm{M}_{i}$ on $\mathbb{R}$ and the existence of an associated pair of well-ordered lower/upper functions $\alpha / \beta$, i.e. $\alpha \leq \beta$ on $[0, T]$. The papers [3] and [13] are based on the method of bound sets, however the only effective criteria contained therein correspond to the situation when there is a well-ordered pair of constant lower and upper functions. In contrast to all these papers, we provide here the existence results which apply also to the case when the pair $\alpha, \beta$ need not be well-ordered and the impulse functions need not be monotone.

Notation. $\mathbb{C}^{1}(J)$ is the set of functions continuously differentiable on the interval $J \subset \mathbb{R}$ and $\mathbb{L}(J)$ is the set of functions which are Lebesgue integrable on $J$. For $u \in \mathbb{L}(J)$, we put $\|u\|_{\infty}=\operatorname{supess}_{t \in[0, T]}|u(t)|$ and $\|u\|_{1}=\int_{0}^{T}|u(s)| \mathrm{d} s$. Furthermore, $\mathrm{D}=\left\{t_{1}, t_{2}, \ldots, t_{p}\right\}, \mathbb{C}_{\mathrm{D}}^{1}$ is the set of functions $u:[0, T] \mapsto \mathbb{R}$ of the form

$$
u(t)=\left\{\begin{array}{l}
u_{[0]}(t) \text { if } t \in\left[0, t_{1}\right], \\
u_{[1]}(t) \text { if } t \in\left(t_{1}, t_{2}\right], \\
\cdots \\
u_{[p]}(t) \text { if } \quad t \in\left(t_{p}, T\right],
\end{array}\right.
$$

where $u_{[i]} \in \mathbb{C}^{1}\left[t_{i}, t_{i+1}\right]$ for $i=0,1, \ldots, p\left(t_{0}=0, t_{p+1}=T\right)$ and $\mathbb{A C}_{\mathrm{D}}^{1}$ denotes the set of functions $u \in \mathbb{C}_{\mathrm{D}}^{1}$ having first derivatives absolutely continuous on each $\left(t_{i}, t_{i+1}\right), i=0,1, \ldots, p$. For $u \in \mathbb{C}_{\mathrm{D}}^{1}$ and $i=1,2, \ldots, p+1$, we define $u^{\prime}\left(t_{i}\right)=u^{\prime}\left(t_{i}-\right), u^{\prime}(0)=u^{\prime}(0+)$ and $\|u\|_{\mathrm{D}}=\|u\|_{\infty}+\left\|u^{\prime}\right\|_{\infty}$. Note that $\mathbb{C}_{\mathrm{D}}^{1}$ is a Banach space with respect to the norm $\|\cdot\|_{\mathrm{D}}$. For $\Omega \subset \mathbb{C}_{\mathrm{D}}^{1}$, $\operatorname{cl}(\Omega)$ and $\partial \Omega$ denote the closure and the boundary of $\Omega$, respectively. If $\Omega$ is open and the operator $\mathrm{F}: \operatorname{cl}(\Omega) \mapsto \mathbb{C}_{\mathrm{D}}^{1}$ is completely continuous and $\mathrm{F} u \neq u$ for all $u \in \partial \Omega$, then $\operatorname{deg}(\mathrm{I}-\mathrm{F}, \Omega)$ denotes the Leray-Schauder topological degree of $\mathrm{I}-\mathrm{F}$ with respect to $\Omega$, where $\mathrm{I}$ is the identity operator on $\mathbb{C}_{\mathrm{D}}^{1}$. If $J \subset[0, T]$, then $\chi_{J}(t)=1$ if $t \in J, \chi_{J}(t)=0$ for $t \in[0, T] \backslash J$. Definitions. A function $u \in \mathbb{A C}_{\mathrm{D}}^{1}$ is a solution of (1.1)-(1.3) if it satisfies the conditions (1.2), (1.3) and fulfils (1.1) a.e. on $[0, T]$.

A function $\sigma \in \mathbb{A C}_{\mathrm{D}}^{1}$ is a lower function of (1.1)-(1.3) if

$$
\begin{aligned}
& \sigma^{\prime \prime}(t) \geq f\left(t, \sigma(t), \sigma^{\prime}(t)\right) \text { for a.e. } t \in[0, T], \\
& \sigma\left(t_{i}+\right)=\mathrm{J}_{i}\left(\sigma\left(t_{i}\right)\right), \sigma^{\prime}\left(t_{i}+\right) \geq \mathrm{M}_{i}\left(\sigma^{\prime}\left(t_{i}\right)\right), i=1,2, \ldots, p, \\
& \sigma(0)=\sigma(T), \sigma^{\prime}(0) \geq \sigma^{\prime}(T),
\end{aligned}
$$


while $\sigma \in \mathbb{A C}_{\mathrm{D}}^{1}$ is an upper function of (1.1)-(1.3) if it satisfies the relations (1.5) but with reversed inequalities.

\section{Green's functions for linear impulsive problems}

For our purposes a proper choice of the operator representation of (1.1)(1.3) is important. To this aim, consider the following linear problem

$$
\begin{aligned}
& u^{\prime \prime}+a_{2}(t) u^{\prime}+a_{1}(t) u=h(t) \text { a.e. on }[0, T] \\
& u\left(t_{i}+\right)-u\left(t_{i}\right)=d_{i}, u^{\prime}\left(t_{i}+\right)-u^{\prime}\left(t_{i}\right)=e_{i}, i=1,2, \ldots, p, \\
& M\left(\begin{array}{c}
u(0) \\
u^{\prime}(0)
\end{array}\right)+N\left(\begin{array}{c}
u(T) \\
u^{\prime}(T)
\end{array}\right)=c,
\end{aligned}
$$

where

$$
\begin{aligned}
& a_{1}, a_{2}, h \in \mathbb{L}[0, T], c \in \mathbb{R}^{2}, d_{i}, e_{i} \in \mathbb{R}, i=1,2, \ldots, p, \\
& M, N \text { are real } 2 \times 2-\text { matrices, } \operatorname{rank}(M, N)=2 .
\end{aligned}
$$

The problem (2.1)-(2.3) can be rewritten as the two-point problem for a very special case of generalized linear differential systems

$$
\begin{aligned}
& x(t)-x(0)-\int_{0}^{t} A(s) x(s) \mathrm{d} s=b(t)-b(0) \text { on }[0, T], \\
& M x(0)+N x(T)=c,
\end{aligned}
$$

where

$$
\begin{aligned}
& x(t)=\left(\begin{array}{c}
u(t) \\
u^{\prime}(t)
\end{array}\right), \quad A(t)=\left(\begin{array}{cc}
0 & 1 \\
-a_{1}(t)-a_{2}(t)
\end{array}\right), \\
& b(t)=\int_{0}^{t}\left(\begin{array}{c}
0 \\
h(s)
\end{array}\right) \mathrm{d} s+\sum_{i=1}^{p}\left(\begin{array}{l}
d_{i} \\
e_{i}
\end{array}\right) \chi_{\left(t_{i}, T\right]}(t), \quad t \in[0, T],
\end{aligned}
$$

and its solutions are in general functions of bounded variation, cf. e.g. [11]. Assume that the homogeneous problem

$$
u^{\prime \prime}+a_{2}(t) u^{\prime}+a_{1}(t) u=0, \quad M\left(\begin{array}{c}
u(0) \\
u^{\prime}(0)
\end{array}\right)+N\left(\begin{array}{c}
u(T) \\
u^{\prime}(T)
\end{array}\right)=0
$$

has only the trivial solution. Then, in view of [12, Theorems 4.2 and 4.3] (see also [10, Theorem 4.1]), the problem (2.5) has the unique solution $x$ and it is given by

$$
x(t)=\int_{0}^{T} G(t, s) \mathrm{d}[b(s)]+x_{0}(t), \quad t \in[0, T],
$$


where $x_{0}$ is the uniquelly determined solution of $x^{\prime}-A(t) x=0$, $M x(0)+N x(T)=c$ and $G(t, s)=\left(g_{i, j}(t, s)\right)_{i, j=1,2}$ is the corresponding Green's matrix. Denote $g(t, s)=g_{1,2}(t, s)$. Then $g(t, s)$ is the Green function of (2.7). Inserting (2.6) into (2.8), taking into account that $\frac{\partial}{\partial s} G(t, s)=-G(t, s) A(s)$, i.e. $g_{1,1}(t, s)=-\frac{\partial}{\partial s} g(t, s)+a_{1}(s) g(t, s)$ (we define $\left.\frac{\partial}{\partial s} g(s, s)=\frac{\partial}{\partial s} g(s-, s)\right)$ and making use of the usual quasilinearization technique, we get the following assertion:

Proposition 2.1. Assume (1.4) and (2.4). Let the problem (2.7) have only the trivial solution and let $g(t, s)$ and $u_{0}$ be respectively its Green's function and the solution of $u^{\prime \prime}+a_{2}(t) u^{\prime}+a_{1}(t) u=0,(2.3)$. Then $u \in \mathbb{A C}_{\mathrm{D}}^{1}$ is a solution to (1.1), (1.2), (2.3) if and only if $u=\mathrm{F} u$, where $\mathrm{F}: \mathbb{C}_{\mathrm{D}}^{1} \mapsto \mathbb{C}_{\mathrm{D}}^{1}$ is the completely continuous operator given by

$$
\begin{aligned}
(\mathrm{F} u)(t) & =\int_{0}^{T} g(t, s)\left(f\left(t, u(s), u^{\prime}(s)\right)-a_{1}(s) u(s)-a_{2}(s) u^{\prime}(s)\right) d s \\
& +\sum_{i=1}^{p}\left(-\frac{\partial}{\partial s} g\left(t, t_{i}\right)+a_{1}(t) g\left(t, t_{i}\right)\right)\left(\mathrm{J}_{i}\left(u\left(t_{i}\right)\right)-u\left(t_{i}\right)\right) \\
& +\sum_{i=1}^{p} g\left(t, t_{i}\right)\left(\mathrm{M}_{i}\left(u^{\prime}\left(t_{i}\right)\right)-u^{\prime}\left(t_{i}\right)\right)+u_{0}(t), \quad t \in[0, T] .
\end{aligned}
$$

\section{Main results}

Our main result is Theorem 3.3 which is the first known existence result for impulsive periodic problems with reversely ordered or non ordered lower and upper functions. In the well-ordered case we have

Theorem 3.1. Let $\alpha$ and $\beta$ be respectively lower and upper functions of (1.1) - (1.3) such that $\alpha \leq \beta$ on $[0, T]$. Assume that (1.4),

$$
\alpha\left(t_{i}\right)<x<\beta\left(t_{i}\right) \Longrightarrow \mathrm{J}_{i}\left(\alpha\left(t_{i}\right)\right)<\mathrm{J}_{i}(x)<\mathrm{J}_{i}\left(\beta\left(t_{i}\right)\right), i=1,2, \ldots, p,
$$

and

$$
\begin{aligned}
& y \leq \alpha^{\prime}\left(t_{i}\right) \Longrightarrow \mathrm{M}_{i}(y) \leq \mathrm{M}_{i}\left(\alpha^{\prime}\left(t_{i}\right)\right), \\
& y \geq \beta^{\prime}\left(t_{i}\right) \Longrightarrow \mathrm{M}_{i}(y) \geq \mathrm{M}_{i}\left(\beta^{\prime}\left(t_{i}\right)\right), \quad i=1,2, \ldots, p,
\end{aligned}
$$

hold. Further, let

$$
\begin{aligned}
|f(t, x, y)| \leq \omega(|y|)(|y|+m(t)) & \\
& \text { for a.e. } t \in[0, T] \text { and all } x \in[\alpha(t), \beta(t)],|y|>1,
\end{aligned}
$$


where $m \in \mathbb{L}[0, T]$ is nonnegative a.e. on $[0, T], \omega \in \mathbb{C}([1, \infty))$ is positive on $[1, \infty)$ and $\int_{1}^{\infty} \frac{d s}{\omega(s)}=\infty$.

Then (1.1)-(1.3) has a solution $u$ such that $\alpha \leq u \leq \beta$ on $[0, T]$.

Notice that our Nagumo type condition (3.3) is weaker than the corresponding assumption by Liz and Nieto [6]. Moreover, we do not assume that $\mathrm{J}_{i}$, $\mathrm{M}_{i}$ are nondecreasing on $\mathbb{R}$. The proof of Theorem 3.1 is based on the topological degree technique. By Proposition 2.1, we can choose a completely continuous operator $\mathrm{F}: \mathbb{C}_{\mathrm{D}}^{1} \mapsto \mathbb{C}_{\mathrm{D}}^{1}$ so that $u$ is a solution to our problem if and only if $u=\mathrm{F} u$. Furthermore, we find $r \in(0, \infty)$ in such a way that $\left\|u^{\prime}\right\|_{\infty}<r$ for each $u \in \mathbb{C}_{\mathrm{D}}^{1}$ which satisfies (1.1) a.e. on $[0, T]$ and is such that $u(t) \in[\alpha(t), \beta(t)]$ on $[0, T]$. If $\alpha<\beta$ on $[0, T]$ and $\alpha(\tau+)<\beta(\tau+)$ for $\tau \in \mathrm{D}$, we can define an open set $\Omega=\Omega(\alpha, \beta, r):=\left\{u \in \mathbb{C}_{\mathrm{D}}^{1}: \alpha<u<\right.$ $\beta$ on $[0, T], \alpha(\tau+)<u(\tau+)<\beta(\tau+)$ on $\left.\mathrm{D},\left\|u^{\prime}\right\|_{\infty}<r\right\}$ and an auxiliary completely continuous operator $\widetilde{\mathrm{F}}: \mathbb{C}_{\mathrm{D}}^{1} \mapsto \mathbb{C}_{\mathrm{D}}^{1}$ so that $\widetilde{\mathrm{F}}=\mathrm{F}$ on $\operatorname{cl}(\Omega)$ and $\widetilde{\mathrm{F}}$ has a fixed point $u \in \mathbb{C}_{\mathrm{D}}^{1}$. To show that this $u$ is a solution of our problem, it suffices to show that $u \in \operatorname{cl}(\Omega)$. To this aim, in the classical case a certain "maximum principle" argument is used. When impulses are present, similar argument can be applied only under the assumptions (3.1) and (3.2) and, surprisingly, only if we rewrite the condition (1.3) in the form $u(0)=u(T)=u(0)+u^{\prime}(0)-u^{\prime}(T)$ and define $\mathrm{F}$ by $(2.9)$, where $u_{0}(t)$ is replaced by $u(0)+u^{\prime}(0)-u^{\prime}(T), a_{1}(t) \equiv a_{2}(t) \equiv 0$ and $g(t, s)$ is the Green function for the Dirichlet problem $u^{\prime \prime}=0, u(0)=u(T)=0$. The extension to the general case with nonstrict inequalities between $\alpha$ and $\beta$ is not difficult. A detailed proof of Theorem 3.1 can be found in [8]. For our further aims, it is important that from this proof also the next assertion on the Leray-Schauder degree of the associated operator I - F follows.

Proposition 3.2. Let the assumptions of Theorem 3.1 be satisfied and let $\alpha<\beta$ on $[0, T]$ and $\alpha(\tau+)<\beta(\tau+)$ on $\mathrm{D}$. Furthermore, let $\mathrm{F}$ and $\Omega(\alpha, \beta, r)$ be defined as above. Then $\operatorname{deg}(I-\mathrm{F}, \Omega(\alpha, \beta, r))=1$

whenever $\mathrm{F} u \neq u$ on $\partial \Omega(\alpha, \beta, r)$ and $r>0$ is sufficiently large.

Theorem 3.3. Let $\alpha$ and $\beta$ be respectively lower and upper functions of (1.1)-(1.3) such that $\alpha(\tau)>\beta(\tau)$ for some $\tau \in[0, T]$ and (3.2) and

$$
\begin{aligned}
& x>\alpha\left(t_{i}\right) \Longrightarrow \mathrm{J}_{i}(x)>\mathrm{J}_{i}\left(\alpha\left(t_{i}\right)\right), \\
& x<\beta\left(t_{i}\right) \Longrightarrow \mathrm{J}_{i}(x)<\mathrm{J}_{i}\left(\beta\left(t_{i}\right)\right), \quad i=1,2, \ldots, p,
\end{aligned}
$$


hold. Further, let $m \in \mathbb{L}[0, T]$ and $|f(t, x, y)| \leq m(t)$ for a.e. $t \in[0, T]$ and all $(x, y) \in \mathbb{R}^{2}$.

Then the problem (1.1)-(1.3) has a solution $u$ satisfying one of the conditions

$$
\begin{aligned}
& u\left(s_{u}\right)<\alpha\left(s_{u}\right) \text { and } u\left(t_{u}\right)>\beta\left(t_{u}\right) \quad \text { for some } s_{u}, t_{u} \in[0, T], \\
& u \geq \alpha \text { on }[0, T] \text { and } \inf _{t \in[0, T]}|u(t)-\alpha(t)|=0, \\
& u \leq \beta \text { on }[0, T] \text { and } \inf _{t \in[0, T]}|u(t)-\beta(t)|=0 .
\end{aligned}
$$

As in the classical case without impulses, the key steps of the proof are: the choice of the operator representation $\mathrm{F}$, • a priori estimates for solutions of a wide family of auxiliary problems, • the choice of a proper auxiliary problem from this family and of the corresponding operator $\widetilde{\mathrm{F}}$, • constructing of "large" well-ordered pair of lower/upper functions, • making use of Proposition 3.2 and of the additivity property of the topological degree to find an open set $\widetilde{\Omega}$ such that $\widetilde{\mathrm{F}} u$ has a fixed point in $\operatorname{cl}(\widetilde{\Omega})$ and, finally, showing that then $u$ is a solution to (1.1)-(1.3). For details see [9].

\section{References}

1. D. Bainov and P. Simeonov, Impulsive Differential Equations: Periodic Solutions and Applications. Longman Sci. Tech., Harlow, 1993.

2. A. Cabada, J. J. Nieto, D. Franco and S. I. Trofimchuk, Dynam. Contin. Discrete Impuls. Systems 7 (2000), 145-158.

3. Dong Yujun, Nonlinear Anal. 27 (1996), 811-820.

4. L. H. Erbe and Liu Xinzhi, J. Math. Anal. Appl. 149 (1990), 56-69.

5. Hu Shouchuan and V. Laksmikantham, Nonlinear Anal. 13 (1989), 75-85.

6. E. Liz and J. J. Nieto, J. Math. Anal. Appl. 161 (1991), 388-394.

7. E. Liz and J. J. Nieto, Comment. Math. Univ. Carolin. 34 (1993), 405-411.

8. I. Rachůnková and M. Tvrdý, Abstr. Appl. Anal., to appear.

9. I. Rachůnková and M. Tvrdý, Mathematical Inst. Czech Acad. Sci., Preprints 151 (2002) and $\mathbf{1 5 3}$ (2003).

10. Š. Schwabik and M. Tvrdý, Czechoslovak Math. J. 29 (104) (1979), 451-477.

11. Š. Schwabik, M. Tvrdý and O. Vejvoda, Differential and Integral Equations: Boundary Value Problems and Adjoint. Academia and D. Reidel, Praha and Dordrecht, 1979.

12. M. Tvrdý, Časopis pěst. mat. 104 (1979), 357-369.

13. Zhang Zhitao, Appl. Math., Ser. B (Engl. Ed.) 12 (1997), 307-320. 